\title{
Detecting Pit Defects on Rail Surface Using A Fast Detection Algorithm Based on Relative Gray Value
}

\author{
Wendi Weng, Houjin Chen \\ School of Electronic and Information Engineering, Beijing Jiaotong University, Beijing, China \\ E-mail: 12120015@bjtu.edu.cn
}

\begin{abstract}
It is a challenge to detect pit defects on rail surface quickly and accurately in machine vision system. As the pit defects appear randomly, vary in size, distribute discontinuously, and are affected by rust, white noise, shadow and illumination during imaging, pit defects detection has become a difficulty in machine vision field. In this paper, we present a fast detection algorithm based on relative gray value to achieve the requirement of detecting defects on rail surface quickly and accurately. This algorithm uses $1 \times N$ dimensional linear mean filtering to improve the detection efficiency. The influence from rust, white noise and environmental impacts are excluded with a set of preprocessing, including offset, contrast of gray values, and image enhancement. Detection accuracy is further improved with Otsu's binary segmentation method. Experimental results show that this algorithm can detect defects on rail surface quickly and accurately.
\end{abstract}

Keywords-Defect on rail surface; Linear Mean Filtering; Relative gray value; Fast algorithm

\section{INTRODUCTION}

Rail transportation has become one of important modern transportations. With the constraints of current manufacturing process, forging rail may produce imperfections including seams, laps, cracks, plug scores, cuts, and gouges [1]. After the continuous crunch from rolling wheels and erosion from the environment, these defects in metal will become a form of chipping, peeling, cracking, scarring, indenting reflected on rail surface. During the process of forging rail, if the rolled steel or oxidized iron is forged onto the head tread of rail, it will also produce small rolling scar defects causing small surface pits on the rail [2]. These uneven rail surface pits become a serious threat to the safety of rail transportation [3]. Thus, it is necessary to adopt an effective method to inspect rail surface carefully and maintained them regularly.

Nowadays, the practical rail defects detecting technology mainly include magnetic detection [3], eddy current flaw detection [4], ultrasonic testing [5], and machine vision inspection [6]. With the advantages of non-contact, high precision and high automation features, the system of using machine vision to detect rail defect has been widely used $[7,8]$. The main difficulty in machine vision detecting method resides in the discrete distributed pits on rail [9], because they appear randomly, differ in sizes, and distribute discontinuously that will eventually lead to a huge volume of work in pits detection. Therefore, there is a need for an efficient method of detecting the defect area to meet the requirements of detecting rail surface defects rapidly. In addition, due to the influence of uneven illumination imaging as well as other environmental factors, the captured images have uneven distributed gray values. What's more, the rust on rail surface and white noise will also have a negative impact on the identification of defect area. Image pre-processing is a key to improve the accuracy of defect detection rate. Adaptive histogram equalization [10] uses gray mean filtering with an $\mathrm{M} \times \mathrm{N}$ window to enhance the contrast of the image which helps to identify the defect area on rail. However, this algorithm consumes large computing capacity and cannot exclude the influence of noise effectively.

\section{RAIL SURFACE IMAGING AND DETECTION ANALYSIS}

In rail surface imaging, part of the surface defects were sunken, leading to a decline of gray values compared with gray values of normal rail. Therefore, a low gray value can be used as a indicator to differentiate defect area and non-defect area. But in practice, most of the surface becomes smooth due to frequent contact with the train wheels; the other part becomes rough due to erosion and other environmental factors, resulting in common rust stripes.

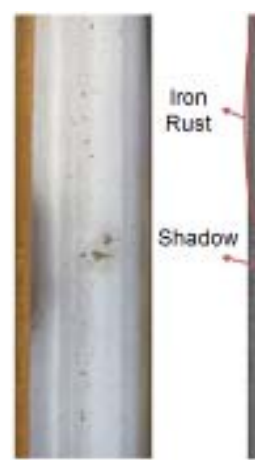

(a)

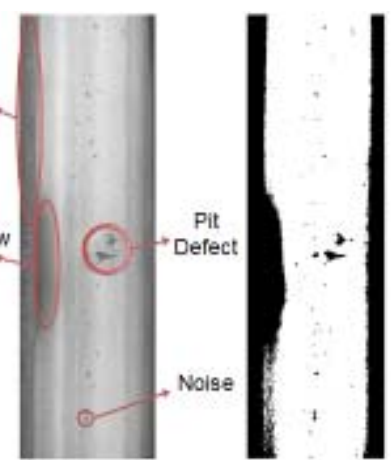

(b)

(c)
Fig. 1. Features of the rail surface: (a) Original rail image (b) Relative gray value image (c) Otsu binary segmentation image

During imaging process, the rough portion of rail surface has low brightness due to diffuse reflection with a relatively low gray value. But this rough area is also part of the rail for normal use, which cannot be taken as rail defects. Because of the different environmental factors and photographing situation, the gray value of same part of rail could differ after 
imaging. From the above analysis, the absolute gray value threshold cannot effectively distinguish the defective part and the non-defective part. Fig. 1(b) shows the absolute gray value image, which contains pit defects, iron rust, shadows, and white noises. The absolute gray value is defined as directly acquired image gray value. Fig. 1(c) shows the Otsu binary segmentation image.

\section{FAst Detection Algorithm Design}

This paper presents a contrast detection fast algorithm as the image preprocessing process, through which the relative gray values of the image would be obtained. Otsu binary segmentation method is used upon relative gray value image to identify the defective area by threshold. In this preprocessing process, linear mean filtering (LMF) is calculating the pixels on the linear space (e.g. a column) rather than the adjacent pixels around it (e.g. the eight pixels around the target pixel as the center) to calculate the average gray value for each pixel. Compared with window mean filtering, linear mean filtering reduces a large volume of computation calculating average gray value without a big trade-off on its accuracy, which is fast and efficient.

\section{A. Preprocessing of Fast Detection Algorithm}

Preprocessing of fast detection algorithm is a key step in calculating the relative gray values of the image and correctly detecting the rail defects. This algorithm includes linear mean filtering, offset, gray value contrast, and image enhancement. The calculation flow is shown in Fig. 2.

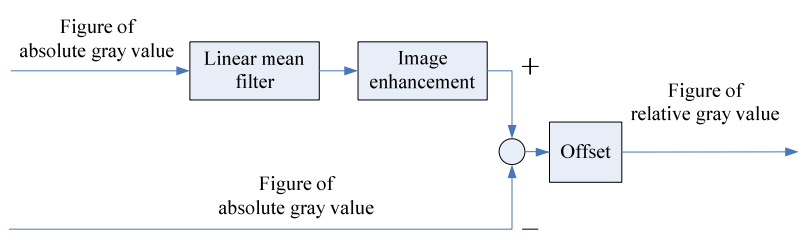

Fig. 2. Preprocessing algorithm flow

As the common rust strip always distribute on both sides of rail edges, the gray values of the imaging is spatially continuous. LMF takes into account the gray value for each pixel of the image and $N$ pixels on the same line. Linear mean computation can effectively eliminate the influence of low gray value from the rail surface rust. However, the image gray values after LMF process are still affected by white noises. Thus, this paper uses offset $\Delta$ to eliminate the influence brought by white noises. In addition, the size of the average value of $\mathrm{N}$ has a significant impact on the result of LMF.

1) Linear mean filtering: First, $y$ axis is defined to be parallel to the train moving direction; $x$ axis is defined to be perpendicular to the train moving direction; $x$ and $y$ coordinate are used to describe the rail surface. The original captured image of rail surface is taken as $I_{x y}$. The image after LMF processing is taken as $L M F_{x y}$, calculated as in:

$$
L M F_{x y}=\sum_{y-(N / 2+1)}^{y+N / 2} I_{x y} / N
$$

Here, $N$ express as the length of the coordinate point $(\mathrm{x}, \mathrm{y})$ on direction of $y$-axis. This advantage of this algorithm is that it can set a reference value for each pixel and when $N$ is large enough, the value of $L M F_{x y}$ will not deviate much because of one or a few points of abnormal gray values.
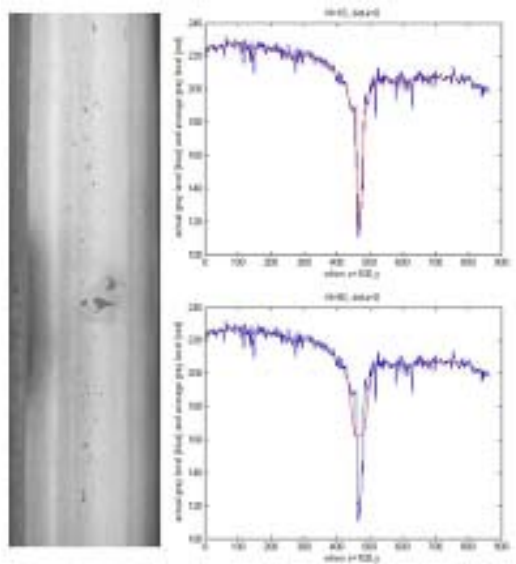

(a)

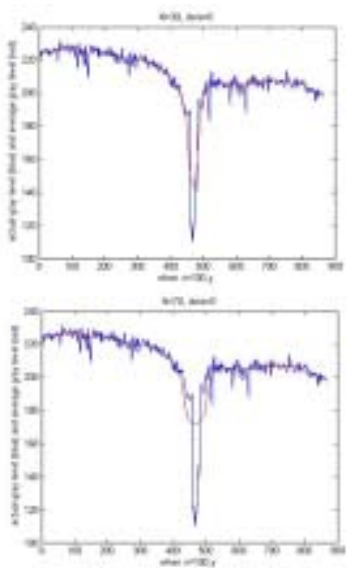

(b)
Fig. 3. When $x=100$, the gray value of $y$ :(a) Actual gray value image (b) The influence of $\mathrm{N}$ for LMF

In production, the value of $N$ is depended on the sampling accuracy. Fig. 3 shows the relation between $N$ value and actual gray value in rail defects detection experiment: In Fig. 3, the left figure is the original rail surface image $(244 \times 863)$; the right four figures show the comparison of the actual gray value (blue) and the average gray value (red) of a single pixel at $(100, y)$. The width of the pit portion of the image is about 20 pixels. As shown in Fig. 3, the difference between actual and average gray value becomes smaller with the incline of $N$. When $N$ is 70, the LMF line (red) declines before the actual line (blue) declines, while LMF line inclines after the actual line inclines. Meanwhile, the declining and inclining range of the LMF line is much smaller than the actual line. Thus, the greater the $N$ value is, the smaller the impact of the defective area will impose on the mean gray value, thereby increasing the accuracy of the calculation upon relative gray value image.

Fig. 3 shows that the gray value of the defect portion is much smaller than its mean gray value. The preprocessing with fast detection algorithm is using this feature to distinguish defect and non-defect rail area.

2) Offset: Only considering those pixels that have smaller gray value than LMF gray value in detecting defects will still be affected by white noise. The actual gray values are fluctuating with the change of $y$. Through the LMF preprocessing, there will be two kinds of areas with low gray values detected: defective area and noise area. This algorithm takes into account of the white noise influence and sets a mean offset $\Delta$, which can help to exclude the uneven gray value influence brought by noises. Namely:

$$
I_{d e l}=L M F_{x y}-\Delta
$$

Here, $I_{d e l}$ is the image after offset; $L M F_{x y}$ is the image after LMF. Theoretically, $\Delta$ value should be slightly greater than 
the standard deviation of $N$ pixels around that point on y-axis, as shown in Fig. 4(a).

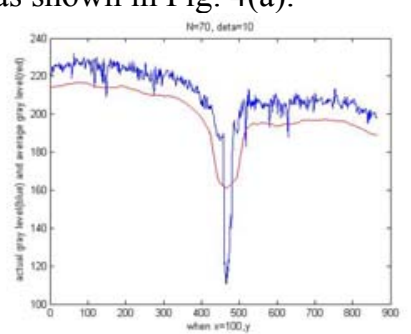

(a)

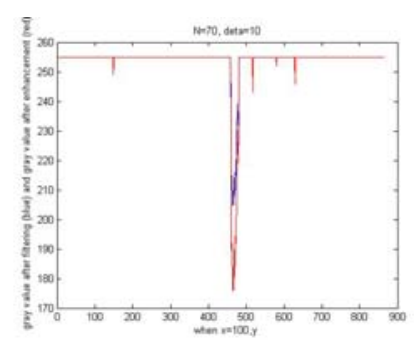

(b)
Fig. 4. (a) Linear average gray value image (b) Image enhancement

3) Gray value contrast: To obtain the relative gray value image, we compare the LMF image $I_{d e l}$ after offset with original gray value image $I_{x y}$. Assuming that relative gray value image is $I_{c o n}$, then:

$$
I_{\text {con }}=I_{\text {del }}-I_{x y}
$$

From formula (3), $I_{\text {con }}$ could be positive, zero, and negative values. A positive value indicates that the gray values of original image is larger than mean ones, which belongs to bright portion and are not in defect area; Zero indicates that the gray values of original image is equal to the mean ones, which belongs to the background and are not in defect area either; A negative value indicates that the gray values of original image is less than the mean ones, which could be in defect rail area.

If taking the shaded background with bright backgrounds together and incorporating them into the background area, the key area could be further strengthened, which will simplify the subsequent image enhancement operations.

$$
I_{L M F}=\left\{\begin{array}{cc}
I_{c o n} & I_{c o n} \geq 0 \\
0 & I_{c o n}<0
\end{array}\right.
$$

4) Image enhancement: From LMF and the relative gray value image operation, $I_{L M F}$ ranges from 0 to $I_{c o n}$. Due to the influence of environmental factors, the background of images collected may be relatively dark. This will limit the range of $I_{L M F}$, which will affect the accuracy of Otsu binary segmentation method. If the range of $I_{L M F}$ is mapped to the gray value range $(0,255)$, it will reduce the environmental impact of a dark background on identifying defect area, as shown in formula (5):

$$
I_{e n h}=255-\left(I_{L M F} \times 255 / I_{c o n}\right)
$$

Here, $I_{e n h}$ is the gray value image after image enhancement, which is shown as the red line in Fig. 4(b). After the image enhancement operation, high gray value area, namely the bright part of the image, is non-defective area; low gray value area, namely the dark part of the image, is where the defects might exist.

5) Analysis of the result of preprocessing: After implementing LMF, offset, gray value contrast, and image enhancement upon original gray value image, we obtain the relative gray value image, as shown in Fig. 5.

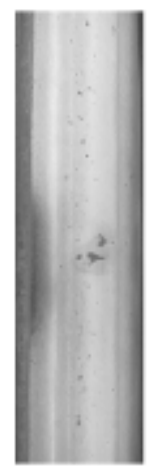

(a)

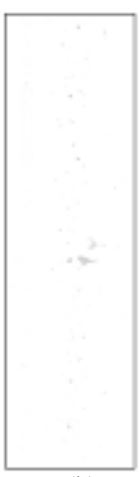

(b)

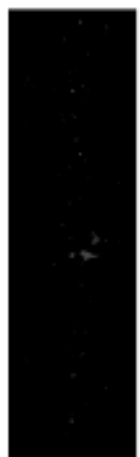

(c)
Fig. 5. Preprogressing result image: (a) Actual gray value image (b) Gray value contrast Image (c) Image after image enhancement

From the obvious results shown in Fig. 5, using a linear space of $1 \times \mathrm{N}$-dimensional LMF operation can quickly and effectively eliminate the influence of rust on identifying rail defects. With an offset and gray value contrast operation, the white noise influence and shadow influence can also be eliminated. In the end, an image enhancement is imposed on the relative gray value image, where the defect area is strengthened and become much more clearly in the image.

\section{B. Rail Defect Localization}

Through the preprocessing algorithm, the image has been divided into background area and area with potential rail defects. Otsu method [11], proposed by a Japanese scholar Nobuyuki Otsu in 1979, is a threshold-based, automated, and unsupervised image segmentation method. Due to its simplicity and effectiveness, this algorithm has aroused wide attention. In this paper, we use the similar idea of the Otsu algorithm to determine the optimal image segmentation threshold by the maximum class variance between background and the objective. This method will divide the image into two parts, foreground and background. The larger class variance between background and the objectives is, the larger difference between the two portions that constitute the image will be. Part of the defect taken as background or part of the background taken as defect will lead to a smaller difference between these two parts. Thus, the use of the maximum class variance between segmentation can reduce the probability of misclassification. By calculating the optimal segmentation threshold, we can determine the probable defect area and obtain the binary segmentation image through the comparison of area's relative gray value and the threshold value.

Considering the continuity of the defect, we use the connected domain filtering method to further identify the defect. By the processing of connected components labeling, we calculate the area of each connected domain and relative gray value and then detect the defect with a judgment between them. Assuming that the threshold of defect area is Thr-area and the defect part of the gray value threshold is Thr-weight. We have formula (6) and (7):

$$
\text { weight }=\sum_{\text {area }} I_{e n h} / \text { area }
$$




$$
\begin{gathered}
\operatorname{Tar}(x)=\text { true; } \\
\text { if }(\text { area }>\text { Thr }- \text { area \& \&weight }>\text { Thr }- \text { weight })
\end{gathered}
$$

Here, $\operatorname{Tar}(x)$ is the target area. When $\operatorname{Tar}(x)$ meets the conditions of area and the gray value requirements, the target area will be determined to be a defect. The result is shown in Fig. 6.

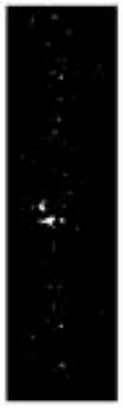

(a)

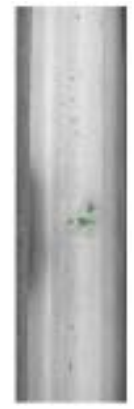

(b)
Fig. 6. Fast detection algorithm results: (a) Otsu binary segmentation map (b) Defect identification through connected domain filtering

\section{EXPERIMENTAL RESULTS AND ANALYSIS}

In order to verify the computing speed and effectiveness of proposed method, this paper collected 364 rail surface images $(x \times y: 244 \times 836)$, which covers the situations of uneven illumination, shadow, etc. that carries out in Matlab. Among the images, 228 images contain a total number of 373 defects. With our fast detection algorithm, the defect recognition rate is $96.78 \%$. One example image of detection result is shown in Fig. 7.

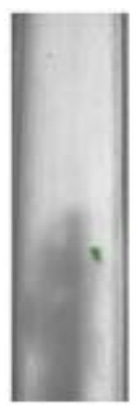

(a)

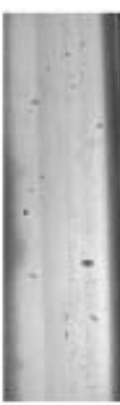

(b)

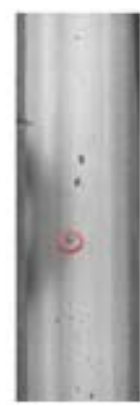

(c)
Fig. 7. Rail defect localization results

The defect area is marked with a green block. The algorithm has strong robustness as it uses threshold determination based on relative gray value image, which excludes the influence of rust and shadow, as shown in Fig. 7(a). In preprocessing algorithm, there is a greater improvement on computing speed with the method of $1 \times \mathrm{N}$ dimensional LMF. Its average detection time is around $30 \%$ faster than the $\mathrm{M} \times \mathrm{N}$-dimensional LMF with a very slight compensation on detection accuracy, which achieves the purpose of fast real-time defects detection, as shown in table I.
TABLE I RaIl Surface Pits Defect Identification Results

\begin{tabular}{ccc}
\hline Algorithm & $\begin{array}{c}\text { LMF processing } \\
\text { average time } \\
\text { (ms/image) }\end{array}$ & $\begin{array}{c}\text { defect } \\
\text { identification rate }\end{array}$ \\
\hline $\mathrm{M} \times \mathrm{N}$ & 60.825 & $98.39 \%$ \\
$1 \times \mathrm{N}$ & 42.274 & $96.78 \%$ \\
\hline
\end{tabular}

\section{SUMMARRY}

To achieve the requirement of fast detection of rail surface defects, we propose a fast detection algorithm. This algorithm calculates the relative gray value of the image from the absolute gray value of the image through a set of operations including $1 \times \mathrm{N}$-dimensional linear mean filtering (LMF), offset, gray value contrast, image enhancement, and Otsu binary segmentation. Experiments show that this algorithm can detect rail surface defects excluding the influence of rust, noise and background with a small amount of computation.

\section{ACKNOWLEDGMENT}

This work was partially supported by National Natural Science Foundation of China (Grant NO. 61271305) and Research Fund for the Doctoral Program of Higher Education of China (Grant NO. 20110009110001)

\section{REFERENCES}

[1] $\mathrm{Hu}$ Yu. Research on Defects Inspection Technology for Rail Surface Base on Machine Vision [D]. Hunan University, 2013

[2] Xiao Qian. The elasto-plastic analysis and fatigue damage research of wheel/rail rolling contact [D]. China academy of railway sciences, 2012

[3] Wu Yi. Effect in Magnetostatic Field-Physics Analysis of Magnetic Powder Detection [J]. Mechanical management and development, 2002 , 4: 49-51

[4] Zhang Liming. Railway Surface Crack Detection Base on Eddy Current [J]. Mechanical Engineer, 2006, 7: 129-130

[5] Hu Jin-ping Ren Jianping. The Application of Ultesonic in Rail Flaw Detection [J]. Shanxi Electronic Technology, 2006, 3: 52-53

[6] Ye Suru, Hu Zhimin, Ouyang Qi, Li Shuxin. The research of the defective detection system of surface of heavy rail on the basis of them machine visual $[\mathrm{J}]$. Moder manufacturing engineering, 2007, 8: 89-93

[7] Wang Ping, Liu Ze, Wang Wei, et al. Rail defect identification using digital image processing and characteristics extraction [J]. Modern Scientific Instruments, 2012(2): 24-28

[8] Wang Haitao, Zhen Li, Yang Chunxia, et al. Rail surface defects detection based on computer vision system $[\mathrm{J}]$. Nondestructive Testing, 2011, 22(11): 38-41

[9] Yuan Xiaocui, Wu Lushen, Chen Huawei. Improved Image Preprocessing Algorithm for Rail Surface Defects Detection [J]. Journal of Computer-Aided Design \& Computer Grephics, 2014, 26(5): 800-805

[10] Tan T L, Sim K S, Tso C P, et al. Contrast enhancement of computed tomography images by adaptive histogram equalization-application for improved ischemic stroke detection[J]. International Journal of Imaging Systems and Technology, 2012, 22(3): 153-160

[11] OTSU N. A threshold selection method form gray level histograms [J]. IEEE Trans on SMC, 1979,9(1): 62-69 